\title{
The Use of Computer-Assisted Diagnosis in Cardiac Perfusion Nuclear Medicine Studies: A Review (Part 2)
}

\author{
Frederick L. Datz, Frank V. Gabor, Paul E. Christian, Grant T. Gullberg, Carol E. Menzel, and \\ Kathryn A. Morton
}

\begin{abstract}
Computer-assisted diagnosis (CAID) is commonly used to evaluate cardiac nuclear medicine studies such as thallium perfusion scans. Part I of this series (Journal of Digital Imaging. Vol 5, No 4, pp 209-222) reviewed the basic theory underlying CAID in nuclear medicine and its use in planar thallium imaging. This review discusses the application of CAID to SPECT perfusion studies.
\end{abstract}

Copyright 1993 by W.B. Saunders Company

$\mathbf{C}$ OMPUTED-ASSISTED diagnosis (CAID) is an everyday part of nuclear medicine. Nowhere is this more true than in cardiac imaging. Most nuclear medicine departments use CAID to calculate ejection fractions, as wêll as other parameters, from radionuclide ventriculograms. ${ }^{1,2}$ At present, the greatest interest in cardiac CAID is for the evaluation of perfusion studies that are performed with thallium and the new technetium agents, sestamibi and teboroxime. ${ }^{3}$

Part I (Joumal of Digital Imaging, Vol 5, No 4, pp 209-222) presented the basic theory of CAID in nuclear medicine and reviewed its use in planar thallium perfusion imaging. This review concentrates on the application of CAID to single-photon emission computed tomography (SPECT).

\section{APPLICATION OF CAID TO SPECT TL-201 IMAGING}

Garcia et al applied the basic CAID techniques that they developed for planar imaging to SPECT studies. ${ }^{4}$ Rather than generating three count profiles, one for each planar view, these investigators created count profiles for selected transaxial and vertical long-axis slices. As in the planar technique, these profiles were compared with a normal file and any pixels that were more than 2.5 standard deviations (or some other criteria) below the mean were flagged as abnormal (Figs 1 and 2). An outline of their SPECT technique is shown in Table 1,5 and a more detailed discussion follows.

\section{Technique}

Quality control is much more important and complex with CAID of SPECT images than it is for planar imaging. ${ }^{5-7}$ Nonuniformity correction, center-of-rotation alignment, pixel size adjustment, head levelness, and other corrections are periodically applied; the adequacy of these corrections must be checked with daily floods and quality control soft-ware (see Artifacts section below for a discussion of the effect of inadequate quality control on CAID).

To assure that the normal file is a valid comparison group, all clinical studies must be acquired with the identical imaging protocol used to generate the normal file. This includes the same injected dose, imaging times postinjection, degree of rotation (eg, $180^{\circ}$ versus $360^{\circ}$ ), number of angles, time of imaging at each stop, and size and number of energy windows. ${ }^{5-8}$ Varying any of these parameters will change the number of counts in the final images independent of the uptake of the tracer in the patient.

The imaging protocol frequently includes a 12 to 15 minute delay between the end of exercise and the onset of image acquisition. ${ }^{9}$ This is in contrast to earlier practice in which Tl-201 imaging was initiated as soon as possible after the termination of exercise. The requirement for rapid imaging was based on a report by Rothendler et al (among others) who did a comparison of early and mildly delayed imaging. ${ }^{10}$ In 42 patients who have proven coronary artery disease, 22 showed transient defects when imaged at 2 minutes and again at 2 hours compared with only 14 defects when imaged at 18 minutes and 2 hours. The differences in results were thought to be due to rapid redistribution into areas of ischemia in patients who have relatively mild degrees of coronary steno-

From the Division of Nuclear Medicine and Medical Imaging Research Laboratory, Department of Radiology, University of Utah School of Medicine, Salt Lake City, UT.

Address reprint requests to Frederick L. Datz, MD, Director, Division of Nuclear Medicine, Department of Radiology, Room IB-647, University of Utah School of Medicine, Salt Lake City, Utah 84132.

Copyright 1993 by W.B. Saunders Company

0897-1889/93/0601-0012\$03.00/0 


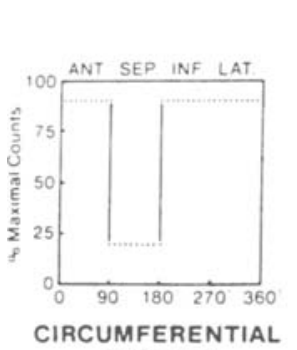
PROFILE

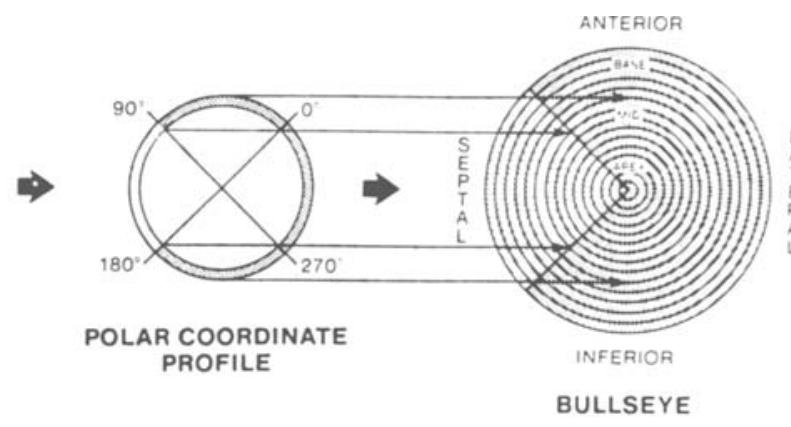

B

C
Fig 1. (A) A circumferential profile is generated for each slice, similar to planar imaging. (B) a polar coordinate profile slice is generated. (C) The profiles are stackec concentrically, with the apical profiles placed centrally and the basal profiles placed peripherally (Reprinted with permission. ${ }^{19}$ ) sis or in those in whom exercise-induced coronary spasm occurs.

A recent report from Friedman et al describing false-positive thallium scans due to "heart creep" has caused the standard imaging protocol to be altered. ${ }^{11}$ The investigators noticed that many normal patients showed reversible inferior and basal inferoseptal defects on SPECT imaging that were due to upward movement of the heart during the image acquisition interval. An increase in the mean total lung volume with deep breathing immediately postexercise causes depression of the heart and diaphragm. As patients gradually catch their breath and reduce the depth of ventilation, the heart and the disphragm move up and assume their normal baseline position. The frequency of heart creep is quite high $-29 \%$ of patients and $39 \%$ of individuals who have less than a $5 \%$ chance of coronary artery disease show artifactual defects. Waiting approximately 15 minutes before beginning imaging greatly reduces the incidence of this phenomenon. The delay has an additional advantage: it allows a planar image to be performed to access lung uptake, a parameter that is difficult to evaluate on SPECT images. ${ }^{12}$

In addition to the imaging protocol, the exercise technique must also be standardized. Treadmill exercise using the Bruce protocol or one of its modifications is the most common technique. The normal file values are based on a maximal study; this is defined by attaining a symptom-limited study (moderately severe anginal pain, severe dyspnea, or severe global fatigue), significant electrocardiogram (EKG) changes ( $4 \mathrm{~mm}$ ST segment depression, malig-

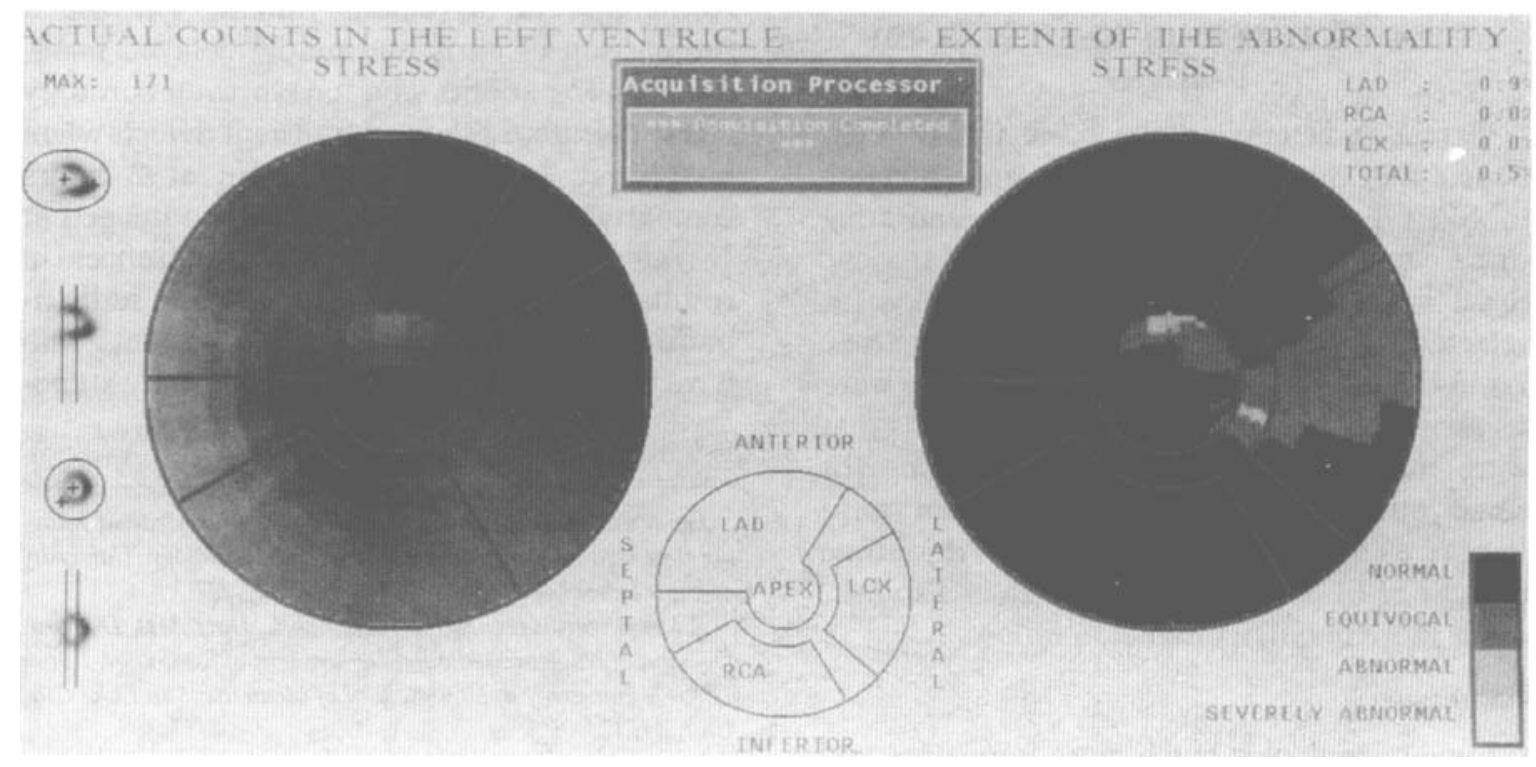

Fig 2. Stress polar plots. On the left are the raw data; on the right, the profiles have been compared with a normal file. 
Table 1. Space/Time Quantitation of SPECT TI-201 Scans

Acquire images using special imaging protocol.

Correct for nonuniformity, center-of-rotation misalignment, etc.

Reconstruct transaxial, sagittal, and oblique tomographic slices using specific filters.

Extract maximal-count profile for selected short- and longaxis slices at stress and redistribution.

Calculate the percent washout for each corresponding slice between stress and redistribution.

Compare the extracted profiles with a normal file.

Map the results onto a two-dimensional display.

Display the extent and severity of perfusion defects.

nant arrythmia), exercise hypotension (greater than $10 \mathrm{~mm} \mathrm{Hg}$ drop between exercise stages), or the achievement of greater than $85 \%$ of the patient's maximal predicted heart rate. ${ }^{9}$ Exercise must be continued for at least 60 seconds after injection of thallium to allow adequate myocardial localization.

It is important that the patient obtain a maximal study because the main determinant of myocardial Tl-201 uptake is the amount of thallium delivered to the heart, which is proportional to the thallium concentration in the blood and the coronary blood flow. ${ }^{13,14}$ Coronary blood flow is dependent on the heart rate obtained. The higher the final heart rate, the more Tl-201 extracted by myocardial tissue. Thus, the average count rates of the normal file would be higher than the count rates from a submaximal clinical study, even if the patient does not have coronary artery disease.

As discussed in Part $\mathrm{I}$, the rate of washout is related to the difference between myocardial and blood concentrations of Tl-201. With a higher initial concentration of Tl-201 in the heart, the washout rate is faster. ${ }^{15}$ For each beat per minute below the maximum predicted heart rate, Tl-201 clearance is slowed by 0.05 hours. Therefore, submaximal exercise studies will be falsely diagnosed as coronary artery disease.

The tomographic reconstruction process must be standardized for each type of camera/ computer. ${ }^{8}$ To select the optimal backprojection filter, each filter's performance is tested by measuring the contrast and uniformity in the reconstructed tomograms of a heart phantom at various cutoffs and orders. The product of the contrast times uniformity is the performance index of the filter at a particular cutoff and order. The filter (with its corresponding parameters) that has the highest performance index for a particular camera/computer system is used.

The data is also filtered in depth. Again, the filter used varies with the system. ${ }^{8}$ The raw data can either be filtered before reconstruction using techniques such as a nine-point weighted smoothing algorithm or a Butterworth lowpass filter, or filtering can be performed on the reconstructed transaxial tomograms using a 1-2-1 smoothing algorithm. One pixel width thickness per slice (approximately $6.2 \mathrm{~mm}$ ) reconstructions are used.

Because transaxial tomograms are frequently normalized so that the maximum reconstructed pixel value does not overflow, a correction must be made to reflect the change in counts between stress and redistribution. ${ }^{4}$ This can be done by using multiplication scale factors provided by the commercial software for each system.

After reconstruction, the long-axis cut with the largest cavity length is chosen to generate the short-axis slices that are used for the polar display. ${ }^{4}$ The three slices closest to the base of the heart and the apex are excluded because of the significant variation in counts in these slices due, in part, to partial-volume effects. Partialvolume effects occur when a portion of the tomographic slice is outside the heart (Fig 3). In addition, by defining the limits of the slices based on the subendocardial aspect of the apex, the same segments can be more accurately compared at stress and redistribution.

On the short-axis slice that is midway between the most apical and basal cuts, the operator defines the center of the ventricular cavity and the search radius. The inferior junction of the right and left ventricle is marked as a reference and is arbitrarily aligned to $102^{\circ} .4$ The final slices are then generated; nine to 12 short-axis and four to six long-axis cuts (Fig 4). The computer then divides the slices into 60 radii of $6^{\circ}$ each and determines the maximum counts in each slice. Final circumferential profiles are then generated in a manner similar to that used in planar imaging (see Part I). Each profile is normalized to the maximum pixel value found in that profile.

After all of the profiles have been created, they are mapped onto a two-dimensional polar 
OBLIQUES

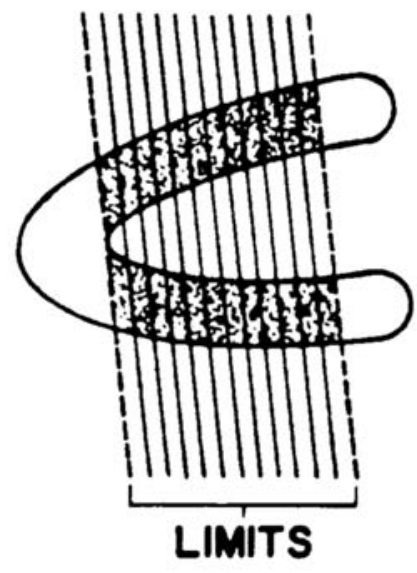

SAGITTALS

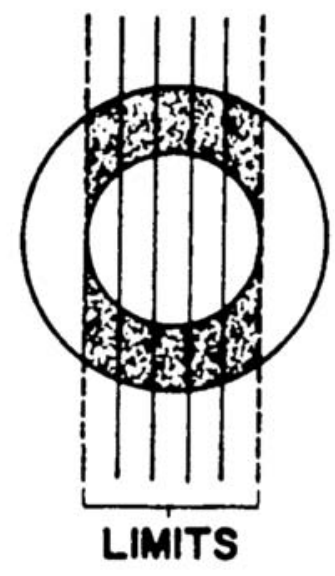

Fig 3. To prevent partial volume artifacts, apex and basal segments are excluded. On shortaxis views, only slices that include the entire ventricle are used, again to prevent partial volume artifacts (Reprinted with permission. ${ }^{4}$ ) representation. Short-axis slices are mapped as concentric circles with the more apical slices on the inside of the display and the more basal slices on the outside. ${ }^{4}$ The apex is specially mapped onto the polar plot. Long-axis slices are used rather than short-axis slices. Arcs between 60 and $120^{\circ}$ of each long-axis cut are mapped onto the center of the display (Fig 5). In Garcia et al's technique, the size of the display is always the same; the size of the ventricle is reflected in the number of circumferential profiles that are mapped. ${ }^{4}$ Thus, the band representing each slice is thinner in larger ventricles than in smaller ones.

A similar two-dimensional plot for washout can also be produced. The percent washout
Fig 4. (A) Each profile is similar to a tomographic slice. (B) Each slice is divided into 6 to $9^{\circ}$ profiles to determine maximum counts. (Reprinted with permission. ${ }^{19}$ )
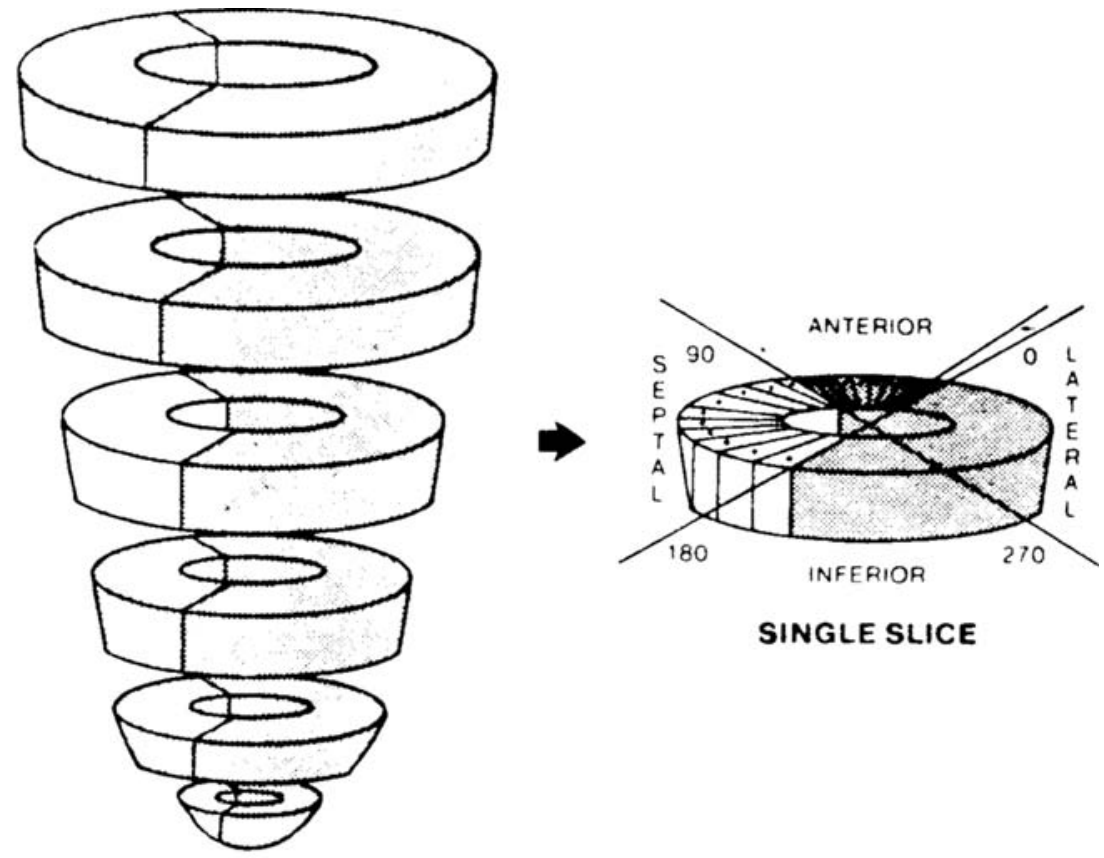

SINGLE SLICE
SHORT AXIS SLICES

A 
Fig 5. An arc between 60 and $120^{\circ}$ on the long-axis is used to map the apex. (Reprinted with permission. ${ }^{4}$ )

circumferential profiles are calculated by subtracting the delayed profile from the stress profile and then multiplying by 100 over the stress profile. ${ }^{4}$ The resultant profile is then mapped onto a two-dimensional polar representation similar to that used for the stress and redistribution plots.

Quantitative analysis is performed on a special set of slices. The ventricle is divided into anatomic regions partly to account for variations in left ventricular size ${ }^{4}$ Five regions, each of equal thickness, are created that represent the volume displayed as the short-axis slices; a second set of five regions are created that represent the volume of the long-axis cuts.

The cases of 20 patients who have a less than $5 \%$ likelihood of coronary artery disease were studied to establish the normal file (see Part I for a discussion using these patients to establish normal profiles). The mean value and standard deviation for each of the 60 angular locations of the ten short- and long-axis regions were obtained by pooling the data of the twenty subjects. Normal limits were then defined by curves that represented 2.5 standard deviations below the mean. ${ }^{4}$ It was found that the number of counts varied significantly based on the anatomic location. Reduced counts were found in the basal portions of the septal and inferior walls due to chest wall and diaphragmatic attenuation. Similarly, the lower limit for washout profiles were defined as 2.5 standard deviations below the mean at 4 hours.

Two types of polar maps, one for the extent of disease, the other for the severity of disease, were generated to determine the best criteria for differentiating normal from abnormal cases. ${ }^{4}$ The extent polar plot is a binary map that sets any points below normal to zero; the remaining points are set to one. The fraction of abnormal myocardium is calculated by determining the percentage of left ventricular pixels that are equal to zero. The severity map displays the abnormal areas as a gray scale, with the level determined by the degree of abnormality. Pixels above normal are given a value of one; values below one are given a fractional value or point score that is linearly dependent on how far it falls below the normal limit.

Vascular territories are assigned as follows $\left(0^{\circ}\right.$ is defined as midseptum with the angles plotted clockwise): 315 to $360^{\circ}$ is assigned to the left circumflex artery; 60 to $120^{\circ}$ represents the right coronary artery; 120 to $315^{\circ}$ is assigned to the left anterior descending artery. ${ }^{4}$

To determine the best criteria to use for diagnosing coronary artery disease, four different techniques have been examined (Table 2).

These criteria were applied to 25 normals and 28 patients who have coronary disease. ${ }^{4}$ The best discriminator between normals and abnormals was the percentage of abnormal pixels at stress. The extent of washout abnormalities or

Table 2. Criteria for an Abnormal Polar Map that were Examined

Extent map (stress)-Percentage of pixels below normal

Extent map (washout)-Percentage of pixels with slow washout

Severity map (stress) - Point score for low uptake

Severity map (washout) Point score for slow washout 
Table 3. Effect of Varying the Percentage of Abnormal Pixels on the Polar Maps

\begin{tabular}{|c|c|c|c|c|c|c|}
\hline \multirow[b]{3}{*}{ Size of abnormality } & \multicolumn{6}{|c|}{ Criteria } \\
\hline & \multicolumn{3}{|c|}{$\begin{array}{l}\text { Extent } \\
\text { Map }(\%)\end{array}$} & \multicolumn{3}{|c|}{$\begin{array}{l}\text { Washout } \\
\text { Map }(\%)\end{array}$} \\
\hline & 10 & 5 & 3 & 10 & 5 & 3 \\
\hline True-positives (\%) & 71 & 82 & 89 & 57 & 64 & 75 \\
\hline True-negatives (\%) & 100 & 100 & 92 & 96 & 96 & 92 \\
\hline
\end{tabular}

the use of the point score did not further discriminate patients who have coronary artery disease from normals. Table 3 shows the effects of varying the percentage of abnormal pixels on the true-positive and true-negative rates.

Based on these results, a $3 \%$ minimum abnormality was chosen as the best trade-off between true-positive and true-negative rates.

The application of similar criteria to the three vascular territories was also performed. ${ }^{4}$ Perfusion defects $5 \%$ or greater of the anterior myocardial region (LAD) and $16 \%$ of the posterior regions (combined RCA and CIRC) were found to give the best tradeoff between truepositives and true-negatives. The larger area needed to diagnose abnormalities in the posterior circulation may be related to the effects of attenuation and the larger standard deviation of counts found in that region.

\section{Results}

Garcia et al studied 25 normals and 28 patients with coronary artery disease using the above program (also referred to as the CedarsSinai program) ${ }^{4}$ Ninety-two percent of normals were correctly classified. The accuracy for diagnosing coronary artery disease using these criteria was $89 \%$. Forty-six of $56(82 \%)$ of abnormal regions were correctly identified.

To determine the interobserver agreement between different operators processing the studies, 10 patients were processed multiple times by two different individuals. Concordance was found for all patients.

The addition of washout data did not improve the detection of coronary artery disease as it has in planar imaging. In planar imaging it appears that washout improves the diagnosis of abnormal segments that are superimposed on normal segments. However, SPECT perfusion images have a contrast improvement as high as 25 to 75 times over planar images. Thus, more cases of coronary artery disease are detected on the routine SPECT stress-redistribution images making washout analysis unnecessary.

Maddahi et al have performed studies to improve image enhancement and to determine optimal parameters for the Cedars-Sinai program. ${ }^{16}$ Phantom studies have been performed to determine the optimal filter for the computerassisted diagnostic program. The filter with the highest efficiency or power product (uniformity $\times$ contrast resolution) was chosen. Maddahi et al have noted that their results apply only to a particular camera/computer system.

Next, these investigators studied 20 normal men (less than 5\% probability of coronary artery disease) and 25 men who have known coronary artery disease to determine the optimal lower limits of normal. After measuring the counts in the normals, they tested three approaches. They developed a lower limit based on a count rate $25 \%$ below the mean normal value for that point. Next they tried a lower limit of 2.5 standard deviations below the mean for each point. Finally, they used a range approach in which the lowest observed value for a point among the 20 normal subjects were used. These three approaches were then prospectively applied to the 20 normals and to 15 patients who had coronary artery disease. All yielded $100 \%$ sensitivity. However, the normalcy rate varied dramatically (Table 4). The distribution of normal profiles was skewed in the anterolateral and inferoseptal regions of the left ventricle and was gaussian in the remaining regions. The standard deviation and $25 \%$ below mean techniques do not account for the skewness of distribution, and therefore did not yield the highest accuracy. Based on the above results, Maddahi et al modified their program to set the lower limit of normal using the range of normal values.

They next looked at the criteria for determining if a perfusion defect is present. Polar maps

Table 4. Results of Three Different Techniques for Determining the Limits of Normal

\begin{tabular}{cccc}
\hline Technique & $\begin{array}{c}-25 \% \\
\langle \%\rangle\end{array}$ & $\begin{array}{c}2.5 \mathrm{SD} \\
(\%)\end{array}$ & $\begin{array}{c}\text { Range } \\
(\%)\end{array}$ \\
\hline Sensitivity & 100 & 100 & 100 \\
Normalcy & 80 & 55 & 100 \\
\hline
\end{tabular}


were divided into two apical regions and 100 segments (five regions from apex to base, each with 20 equidistant sectors). Patients who had known coronary artery disease were studied. For each of the 102 segments, the probability that segment represented a given coronary artery distribution was defined by the frequency with which the segment showed a perfusion defect when known disease in the corresponding coronary artery was present. The final area of each vascular territory was defined as all segments that had an $80 \%$ or greater probability of being abnormal when coronary artery disease was present in the corresponding vessel. Using this approach, there are areas in which no vascular territory is assigned, such as between two contiguous territories. This prevents the incorrect assignment of abnormalities to the wrong vessel and also prevents large defects from extending into a second vascular territory and being misdiagnosed as two-vessel disease (Fig 6).

Receiver operating characteristic curves were then developed to determine how much of each vascular distribution had to be abnormal to be considered a definite defect. The optimal criterion for an abnormality in the left anterior descending artery and left circumflex coronary artery distributions was $12 \%$ or greater, and $8 \%$

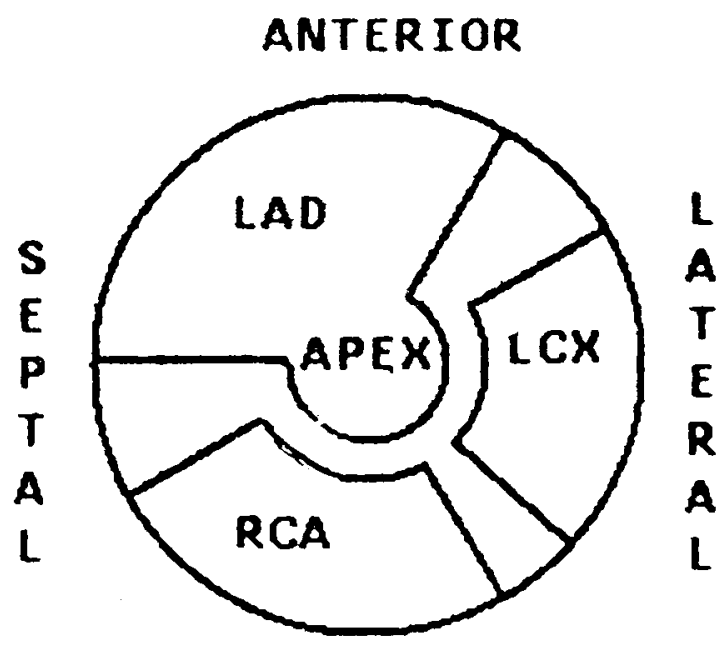

INEERIOR

Fig 6. Vascular distribution using a polar plot; some areas are not assigned to any vascular distribution to prevent misdiagnosis. LAD, anterior descending artery; LCX, left circumflex; RCA, right coronary artery.
Table 5. Accuracy of Criteria for Perfusion Abnormalities

\begin{tabular}{cccc}
\hline & LAD & CIRC & RCA \\
& $(\%)$ & $(\%)$ & $(\%)$ \\
\hline True-positive & 74 & 75 & 76 \\
True-negative & 83 & 78 & 71 \\
\hline
\end{tabular}

Abbreviations: LAD, anterior descending artery; CIRC, circumflex; RCA, right coronary artery.

or greater for the right coronary artery distribution. Use of these criteria yielded true-positive and true-negative rates as outlined in Table 5.

Overall coronary artery disease was defined as being present if at least one of the vascular territories met the criteria of having a perfusion defect present. When tested on a pilot group, these criteria yielded $100 \%$ sensitivity for the detection of coronary artery disease. The application of a probabilistic approach may be more helpful in defining the overall likelihood of coronary artery disease. The larger and more severe a defect, the more likely it is to be a true defect. Further investigation is needed to test this hypothesis.

All the above criteria were applied prospectively to a group of 92 patients who had angiographically confirmed coronary artery disease. The sensitivity for detection of coronary artery disease was $95 \%$ (87/92). Of 43 patients who had prior myocardial infarction, the sensitivity was $100 \%$ regardless of the number of diseased vessels. In the 49 patients who did not have prior infarction, the sensitivity for single-, double-, and triple-vessel disease was $70 \%(7 / 10)$, $94 \%(16 / 17)$, and $95 \%(21 / 22)$, respectively. Of 18 patients who had normal angiograms, the specificity was $56 \%(10 / 18)$. Of 28 patients who had a low likelihood of coronary artery disease, the normalcy rate was $86 \%(24 / 28)$. The results are summarized in Table 6.

The sensitivity for disease varies by the involved vessel. In 138 patients studied prospectively, the sensitivity and specificity by vessel are shown in Table 7.

Table 6. Sensitivity in Patients Who Have Angiographically Positive Coronary Artery Disease

\begin{tabular}{lcccc}
\hline & $\begin{array}{c}\text { Overall } \\
(\%)\end{array}$ & $\begin{array}{c}\text { Single } V \\
(\%)\end{array}$ & $\begin{array}{c}\text { Double } V \\
(\%)\end{array}$ & $\begin{array}{c}\text { Triple V } \\
(\%)\end{array}$ \\
\hline $\begin{array}{c}\text { Prior myocardial } \\
\text { infarction }\end{array}$ & 100 & 100 & 100 & 100 \\
$\begin{array}{c}\text { No prior myocardial } \\
\text { infarction }\end{array}$ & 95 & 70 & 94 & 95 \\
\hline
\end{tabular}


Table 7. Sensitivity and Specificity by Vessel

\begin{tabular}{cccc}
\hline & $\begin{array}{c}\text { LAD } \\
(\%)\end{array}$ & $\begin{array}{c}\text { CIRC } \\
(\%)\end{array}$ & $\begin{array}{c}\text { RCA } \\
(\%)\end{array}$ \\
\hline Sensitivity & 78 & 79 & 82 \\
Specificity & 85 & 60 & 71 \\
\hline
\end{tabular}

The above program has been modified by adding gender-matched normal files. ${ }^{8}$ The cases of 15 normal females were added to the database. Only the appropriate gender-matched group for each patient was used as the normal file (ie, either 20 normal males or 15 normal females).

As noted in Part I, there are differences in the count distribution based on gender (Fig 7). Eisner et al studied 50 males and 50 females with a less than $5 \%$ probability of coronary disease using the bull's-eye display. ${ }^{17}$ Their findings are outlined in Tables 8 and 9 .

There is more variation in count activity in the heart than would be expected. Animal studies indicate that there are only minor differences in blood flow to different regions of the left ventricle as measured by radiolabeled microspheres. ${ }^{18}$ Therefore, it is presumed that the differences in activity are primarily due to soft tissue attenuation. In females, this is breast tissue; in males, it appears to be the diaphragm. ${ }^{17}$

This modified program has been tested in a multi-institutional trial involving 10 sites and more than 318 patients, and compared with results in 168 patients at Cedars-Sinai Medical Center. ${ }^{8}$ In this study, the overall sensitivity for coronary artery disease was $94 \%$ in the multicenter group and $95 \%$ at Cedars; the normalcy rate was $83 \%$ versus $75 \%$. There were no
Table 8. Variations in Polar Plots for Males

All septal segments had decreased counts when compared with the corresponding lateral wall segment.

All inferior segments had decreased counts when compared with the corresponding anterior wall segment.

The apex had decreased counts when compared with adjacent septal, anterior, and lateral wall segments, but approximately the same counts as the adjacent inferior wall segment.

Inferior wall counts on stress images were $7.1 \%-13.2 \%$ less than in females.

differences in results based on the different camera/computer combinations used at the various sites. There were also no differences in accuracy based on the patient's gender (Fig 8).

DePasquale et al have tested a CAID program that is similar to the above program with several important differences. ${ }^{19}$ First, the apical portion of the map is derived from the shortaxis slices rather than from the vertical long-axis slices. Second, normalization of each pixel is the most normal region of the bull's-eye, rather than to each slice. Third, all three sets of orthogonal images are used for quantitative analysis instead of just the short- and vertical long-axis slices. All the short-axis slices are quantified; the most basal three slices are not excluded. The verticle is divided into 40 sectors of 9 degrees each. Standard deviation is used to define the lower limit of normal. A clustering criterion is used; two adjacent pixels must be abnormal for any one pixel to be flagged as abnormal. This results in defects being defined with smoother boundaries. At least $1.5 \%$ of
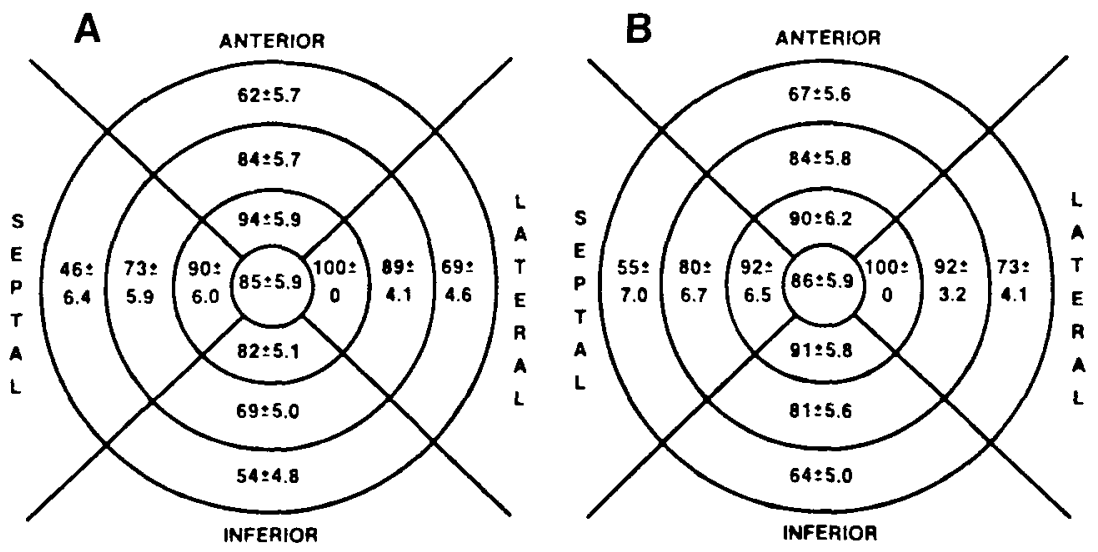

Fig 7. Stress bull's-eye plots showing variation in counts, (A) male versus (B) female (Reprinted with permission. ${ }^{31}$ ) 
Table 9. Variations in Polar Plots for Females

As for males, all septal segments had decreased counts when compared with the corresponding lateral wall segment.

In contrast to males, all inferior wall segments had counts that were similar to the corresponding anterior wall segment.

The apex had decreased counts when compared with the adjacent septal, anterior, and lateral wall segments. Unlike males, the apex also showed decreased counts when compared with the adjacent inferior wall segment.

Anterior wall counts were $4.8 \%-9.2 \%$ less than in males

Washout rates were faster than males by $5 \%$.

The female plot showed less segmental variation than the male plot.

pixels in men and $3 \%$ in women have to be abnormal to diagnose coronary artery disease. Washout analysis is used.

DePasquale et al compared their results with quantitative analysis to that obtained by visual analysis. The results of a prospective study of 210 patients are summarized in Table 10. The results for single-, double-, and triple-vessel disease are shown in Table 11. The results in individual vessels are given in Table 12.

\section{Problems With the Technique}

Tamaki et al and others have shown that SPECT is superior to planar imaging for the measurement of infarct volume. ${ }^{20}$ The determination of the percentage of abnormal myocardium is not completely accurate, however, because of cardiac and thoracic motion. ${ }^{4}$ This motion causes the overestimation of defect activity when infarct size or myocardial blood flow are quantified. ${ }^{21,22}$ Motion artifacts such as these can also result in false-negative results, especially in patients who have small hearts with high ejection fractions or those who have hypertrophy. ${ }^{23}$ In addition, false-positive results have also been reported in cases of patients who have large hyperdynamic hearts and those who have cardiomyopathy. Surprisingly, the distortion caused by motion is not considered as great a problem as scatter and attenuation. ${ }^{4}$

The accuracy of the percentage of abnormal myocardium is also affected by the system's spatial resolution. There is a significant reduc- tion in the reconstructed voxel value for objects smaller than twice the full width at halfmaximum of the spatial resolution, with the effect increasing as the object size falls. ${ }^{24}$ This effect has been seen with infarct sizing and measuring blood flow. ${ }^{21,22}$ Thus small nontransmural defects are missed whereas the size of transmural defects may be overestimated.

The Garcia et al technique gives equal weight to the contribution of both small and large slices. ${ }^{4}$ To accurately measure the size of perfusion defects, the differences in myocardial mass contributed by slices of different sizes and endocardial-to-epicardial thickness need to be accounted for. In addition, the three basal slices are not used; these would need to be included.

Attenuation is a significant problem with Tl-201 because of its low energy mercury $\mathrm{K}$ characteristic $\mathrm{x}$-rays in the 69 to $83 \mathrm{keV}$ range. The half-value thickness for Tl-201 is $3.7 \mathrm{~cm}$ of soft tissue compared with $4.6 \mathrm{~cm}$ for Tc- $-99 \mathrm{~m} .{ }^{3}$ An example of the problems of attenuation are discussed in the Artifacts section, below. Attenuation can be corrected by a number of techniques. One of the most promising is the use of simultaneous transmission emission tomography, ${ }^{3,25}$ Using a triple-headed camera, a technetium source is connected to the gantry so that a transmission map can be obtained at the same time the other two heads are obtaining the emission thallium data. By applying the transmission map to correct the images for attenuation, a truer representation of the distribution of thallium can be obtained. These techniques are not readily available at present, however.

The detection of single-vessel disease is relatively low in some studies. ${ }^{4}$ However, increasing sensitivity for coronary artery disease as the number of diseased vessels increases has been observed in the visual detection of atherosclerotic heart disease as well. ${ }^{26}$

A number of factors may contribute to the number of false-positive and false-negative studies that are not directly related to the technique itself. Coronary angiography is an imperfect gold standard. The variability in interpretation of coronary arteriograms is quite high, limiting their utility as an objective standard. ${ }^{27,28}$ In addition, coronary arteriography is an anatomic study whereas Tl-201 imaging is a physiological study. Even in accurately performed, the mea- 


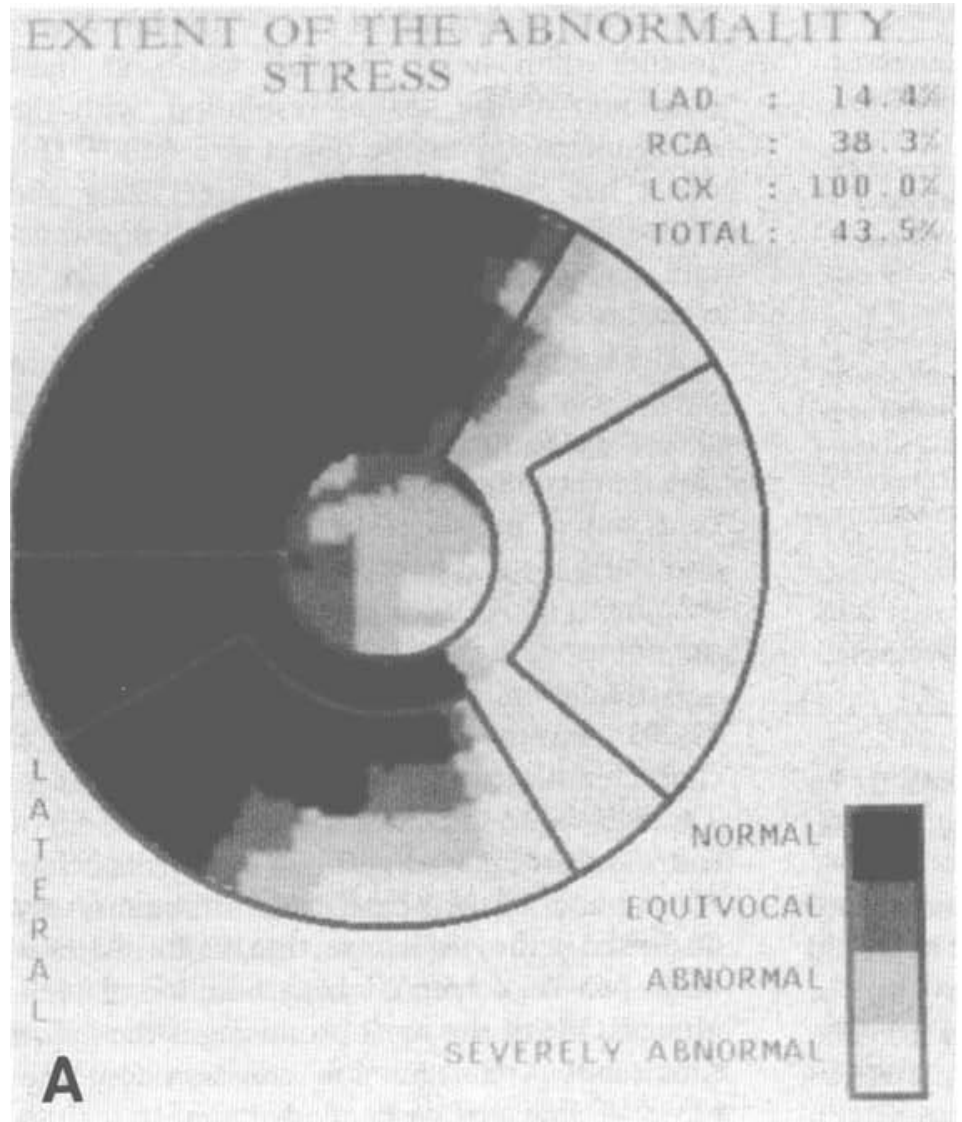

EXIENT OF THE ABNORMALIT Y REST

$$
\begin{aligned}
& \text { LAD }: \quad 0.0 \% \\
& \text { RCA }: \quad 0.0 \%
\end{aligned}
$$$$
\text { LCX : } 0.0 \%
$$$$
\text { TOTAL: } \quad 0.0 \%
$$

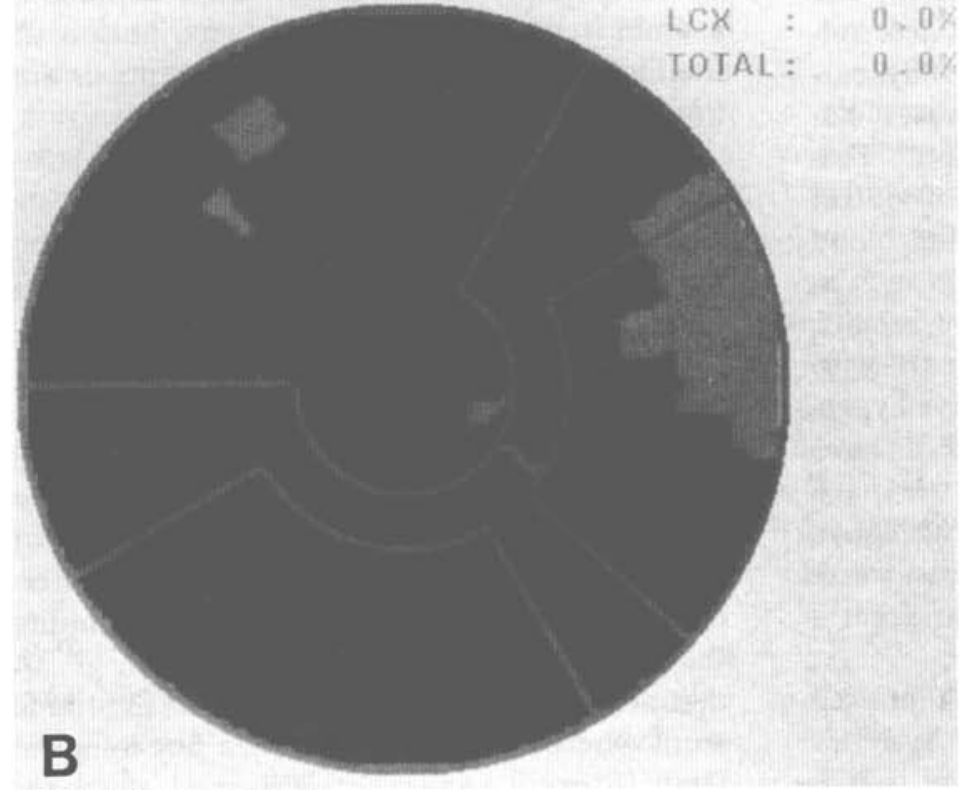

Fig 8. (A) Stress polar plot showing significant defects primarily in the circumflex and right coronary artery circulation. (B) Filling in of the defects occurs at redistribution, indicating ischemia. 
Table 10. DePasquale Results for the Detection of Coronary

\begin{tabular}{lccc}
\multicolumn{4}{c}{ Artery Disease } \\
\hline & $\begin{array}{c}\text { Visual } \\
(\%)\end{array}$ & $\begin{array}{c}\text { CAID } \\
(\%)\end{array}$ & $\begin{array}{c}\text { Combined } \\
(\%)\end{array}$ \\
\hline Sensitivity & 97 & 95 & 95 \\
Specificity & 68 & 74 & 71 \\
\hline
\end{tabular}

surement of diameter of stenosis may not accurately reflect the flow poststenosis. Other factors of the lesion such as the length, shape, eccentricity, and number of lesions in series may also affect blood flow. ${ }^{29}$ The measurement of coronary flow reserve may help to reduce these problems, but is difficult to perform in these types of studies. ${ }^{19}$

Another problem is the variation in normal coronary artery anatomy. A common problem found by DePasquale et al was a defect in the right coronary artery distribution produced by circumflex lesions. ${ }^{19}$ By combining the right coronary and circumflex arteries into a single zone, the sensitivity and specificity could be significantly improved.

False-negatives can occur if the myocardium supplied by a diseased vessel does not become ischemic. This may occur in multivessel disease because the patient's exercise is limited by ischemia in the more diseased vessel. The use of dipyridamole stress can prevent this problem. $3,19,30$

Other causes of false-positives are discussed in the Artifact section, below.

\section{ARTIFACTS}

There are a number of causes of artifacts on SPECT imaging and CAID that can produce false-positive studies. The causes are outlined in Table 13 and discussed in more detail below.

\section{Quality Control Problems}

High count flood images of 30 to 45 million counts must be performed to correct nonuniformities in the field. If nonuniformities are present, ring artifacts can occur. We (and others) have seen similar artifacts due to intermittent

Table 11: DePasquale Sensitivity for Coronary Artery Disease by Number of Diseased Vessels

\begin{tabular}{cccc}
\hline & $\begin{array}{c}\text { Single } \\
(\%)\end{array}$ & $\begin{array}{c}\text { Double } \\
(\%)\end{array}$ & $\begin{array}{c}\text { Triple } \\
(\%)\end{array}$ \\
\hline Sensitivity & 91 & 99 & 100 \\
\hline
\end{tabular}

Table 12. DePasquale Sensitivity for Coronary Disease in Individual Vessels

\begin{tabular}{llll}
\hline & $\begin{array}{c}\text { LAD } \\
(\%)\end{array}$ & $\begin{array}{c}\text { CIRC } \\
(\%)\end{array}$ & $\begin{array}{c}\text { RCA } \\
(\%)\end{array}$ \\
\hline CAID & 78 & 89 & 65 \\
Visual & 70 & 88 & 50 \\
Combined & 75 & 87 & 55 \\
\hline
\end{tabular}

Abbreviations: $L A D$, anterior descending artery; $C I R C$; $R C A$, right coronary artery.

failure of circuit boards. Because of the low counts found in a typical SPECT image, nonuniformities are often hidden in the statistical noise. If present, they indicate a major detector nonuniformity problem. ${ }^{7}$

Errors in the center of rotation will also cause artifacts. If the offset is in the positive (rightward) direction, defects occur in the posteroapical region; if the offset is in the negative direction, defects occur in the anteroapical region. In horizontal long-axis slices, the artifacts appear linear with the anterior and posterior walls misaligned. In bull's-eye plots, the artifacts are comma-shaped.

\section{Patient Motion}

Patients may stretch their arms or move their body during a SPECT study; this results in enough vertical motion to create an artifact. In a typical imaging department, patient motion that is significant enough to cause an artifact on SPECT occurs in $10 \%$ of studies. ${ }^{31}$

Eisner has studied the effects of patient motion on SPECT images. ${ }^{31} \mathrm{He}$ found that vertical motion of as little as 0.5 pixels $(3 \mathrm{~mm})$ can cause an artifactual abnormality on the bull's-eye display. Patient motion of 1 pixel or more causes an abnormality in $40 \%$ of polar plots.

Several investigators have written motion detection and correction algorithms. A cross-

Table 13. Causes of TI-201 Artifacts

\footnotetext{
Quality control problems (nonuniformity and center of rotation errors)

Patient motion

Reconstruction and polar plot errors

Soft-tissue attenuation (breast, diaphragm, chest wall fat)

Overlying visceral activity

Myocardial hot spots

Apical anatomic variations

Noncoronary artery disease (LBBB, hypertrophy, devorotation, and levorotation)
} 
correlation algorithm that detects abrupt motion in the vertical direction only has been developed. ${ }^{31}$ Frame-to-frame pixel shift that deviates beyond an established threshold from a parabolically fit distribution profile is assumed to be motion and is shifted. This method does not always work on redistribution images because of their lower count rate. A second approach uses a diverging squares algorithm that attempts to track the center of the left ventricle and detect motion in two directions. ${ }^{32}$ This is done by expanding a box placed iteractively in the left ventricle in the direction of maximal counts.

For motion detection and correction software to be successful, it must detect motion in all directions, detect both abrupt and gradual movement, and work on both stress and redistribution images. ${ }^{19}$ At present, no software exists that meets all these criteria.

\section{Reconstruction and Polar Plot Errors}

If the long-axis of the left ventricle is incorrectly drawn, the geometry of the reconstructed orthogonal slices may be distorted. ${ }^{7}$ Such mistakes usually result in polar plots that have areas of artifactually decreased counts in the basal myocardial rings at the periphery of the plot and in the apical region (Fig 9).

The count density of the apex may be over- or underestimated in methods that reconstruct the
A

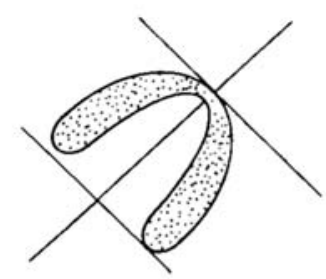

B

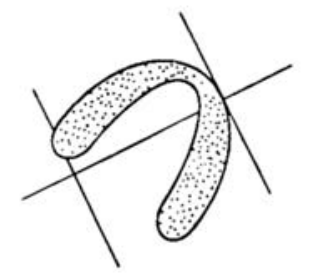

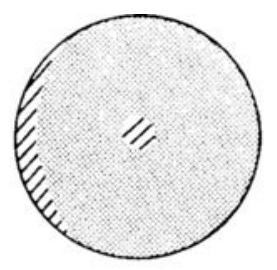

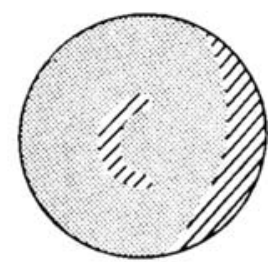

Fig 9. (A) Correct selection of a long-axis results in a bull'seye with regions of physiologically decreased counts (crosshatched areas) at the upper septum and apex. (B) With incorrect selection of the long-axis, defects are produced in the periphery of the plot and in the apical region. (Reprinted with permission.') apex from short-axis slices. In these cases, if the operator places the apical limit too far beyond the actual apex, a partial volume effect will occur. This results in artifactually lower counts. If the apex limit is marked too far toward the base of the heart as can occur in patients who have true apical defects, the extent and severity of the defect will be underestimated on the polar plot.

\section{Soft Tissue Attenuation}

Although gender-based normal files are used, women with large or dense breasts will have marked anterior wall attenuation that will be flagged as abnormal by the program. For example, the Cedars program is not accurate for women with a brassiere size larger than B cup. ${ }^{6}$

The location of a breast defect can vary. Women who have firm breasts that lie on the anterior chest wall when supine will show a defect over the anterior wall. ${ }^{7}$ Women who have pendulous breasts that lie adjacent to the lateral wall when supine will show lateral wall defects. If the patient is imaged with her brassiere on, the breasts will appear thicker and the defects will be more marked. In women who have breast implants, the anterior attenuation will appear high and the defects will be especially marked.

Women who have very small breasts, or those who have undergone mastectomy have little anterior wall attenuation. These patients will show a typical male count density distribution. This causes the inferior wall to be flagged because this area will appear abnormally decreased.

Breast artifacts can occur in men with gynecomastia. In these cases an artifactual defect will be flagged in the anterior wall.

Obese patients may have a lateral chest wall fat pad. These patients will show a lateral wall perfusion defect.

Normally there are decreased counts in the inferior wall compared with the anterior wall, especially in men. However, if abdominal protuberance or left hemidiagraphm elevation is present, increased attenuation of counts occurs. This phenomenon has been described in patients who have ascites secondary to peritoneal dialysis. ${ }^{33}$

Most cases of soft tissue attenuation cause a fixed defect that mimics a myocardial infarction. 
However, if female patients are imaged at one setting their their brassiere off and at another with their brassiere on, they will appear to have a reversible defect due to differences in the location of the breast attenuation. ${ }^{7}$

\section{Overlying Abdominal Visceral Activity}

Patients are NPO for perfusion studies to reduce the amount of tracer accumulation in the abdomen. However, if the stress level achieved is submaximal, or if the study is done with pharmacologic stress, increased activity will occur in the abdomen. If these patients have elevation or eventration of the left hemidiaphragm, the visceral activity may be superimposed on the inferior wall of the left ventricle.? Because some polar plots are normalized to the region of the myocardium with the greatest count density, the remainder of the myocardium will appear to have relatively decreased counts. Visceral activity increases on redistribution images; therefore, these types of artifacts are more likely to be seen on the delayed images. This can lead to the false appearance of reverse redistribution. With dipyridamole stress, visceral activity is usually higher on the immediate images, mimicking ischemia. Inspection of the planar projection data set will allow these artifacts to be identified.

\section{Myocardial Hot Spots}

Focal areas of increased activity are occasionally present in the region of the papillary muscles caused by increased myocardial thickness. ${ }^{7}$ These hot spots are especially common in patients with left ventricular hypertrophy. However, some hot spots are seen in areas that are clearly away from the papillary muscles. The cause of these hot spots is not known. Although they can be present on both stress and redistribution images, they are somewhat more common on stress views. Thus there can be change between stress and redistribution images that mimics ischemia.

Hot spots cause a normalization problem similar to overlying visceral activity and create large artifactual areas of decreased activity. The reconstructed images must be reviewed to spot these types of artifacts. It is very difficult to appreciate hot spot artifacts by only viewing the polar plot images.

\section{Apical Variations}

Some patients show very prominent apical thinning. If this occurs at the very tip of the apex on the horizontal long-axis views, it is usually not a problem for CAID programs. However, if the apical defect is displaced laterally or septally, it can create a defect that will be interpreted by the program as abnormal. ${ }^{7}$ Inspection of the reconstructed horizontal long axis cuts will prevent a false-positive diagnosis.

\section{Noncoronary Disease}

In patients who have ventricular hypertrophy such as from longstanding hypertension and valvular heart disease, a relative decrease in lateral wall counts can occur. In a study of normotensive controls versus hypertensive patients, the lateral-to-septal count density ratio was 1.02 versus $1.17 .{ }^{34}$ The relative decrease in lateral wall activity is present on both stress and redistribution images, mimicking infarction. The patient's history and the EKG findings can be helpful, although it should be noted that the EKG is relatively insensitive for the diagnosis of hypertrophy.

Left bundle branch block (LBBB) can cause false-positive studies. Animal experiments have shown LBBB results in decreased blood flow to the interventricular septum. ${ }^{29}$ The decreased flow is likely the result of asynchronous relaxation of the septum which is out of phase with diastolic filling for the rest of the ventricle; coronary blood flow is maximal during diastolic filling. In a study of 10 patients who have LBBB and normal coronary angiograms, $90 \%$ showed reversible septal defects on Tl-201 imaging. ${ }^{35}$ The changes were most marked in patients who achieved high heart rates; septal asynchrony is less marked at lower heart rates.

Patients who have cardiac rotation will also have an altered lateral-to-septal count ratio. ${ }^{7}$ This artifact is seen in patients who have congenital heart disease, marked selective dilation on one ventricle, and hyperexpansion of the lungs. If the heart is dextrorotated, the septum lies closer to the camera in the right anterior oblique and anterior projections; the lateral wall lies further from the detector in the lateral and left posterior projections. This results in an apparent increase in septal wall count density and a decrease in lateral wall count density. The 
defect is fixed and mimics a myocardial infarction. In contrast, with levorotation there is an apparent fixed septal defect. Inspection of the transaxial slices and the EKG will allow variations in cardiac orientation to be determined.

\section{CONCLUSION}

The use of quantitative analysis for SPECT studies can be helpful in the diagnosis of coro- nary artery disease. However, there are a number of technical and physiological artifacts that can significantly reduce the utility of CAID if they are not excluded before accepting the computer interpretation of each study.

In the third part of this series, we will look at new types of polar displays and the application of expert systems and neural networks to the diagnosis of coronary artery disease.

\section{REFERENCES}

1. Christian PE, Nortmann CA, Taylor AT: Comparison of fully automated and manual ejection fraction calculations: Validation and pitfalls. J Nucl Med 26:775-782, 1985

2. Niemann $H$, Bunke $H$, Hofmann I, et al: A knowledgebased system for analysis of gated blood pool studies. IEEE Trans Pattern Anal Mach Intell 7:246-259, 1985

3. Datz FL, Gullberg GT, Gabor FV, et al: SPECT myocardial perfusion imaging update. Semin Ultrasound CT MRI 12:28-44, 1992

4. Garcia EV, VanTrain K. Maddahi J, et al: Quantification of rotational thallium-201 myocardial tomography. J Nucl Med 26:17-26, 1985

5. DePuey EG, Garcia EV, Ezquerra NF: Three-dimensional techniques and artificial intelligence in thallium-201 cardiac imaging. AJR Am I Roentgenol 152:1161-1168, 1989

6. Gabor FV, Datz FL, Christian PE, et al: Computerassisted diagnosis of cardiac perfusion studies. J Nucl Med Technol 19:238-244, 1991

7. DePuey EG, Garcia EV: Optimal specificity of thallium-201 SPECT through recognition of imaging artifacts. $J$ Nucl Med 30:441-449, 1989

8. VanTrain KF, Maddahi J, Berman DS, et al: Quantitative analysis of tomographic stress thallium-20I myocardial scintigrams: A multicenter trial. J Nucl Med 31:1168-1179, 1990

9. Cedars-Sinai PTQ: Nuclear Medical Imaging System Operator's Guide. Bedford Heights, OH, Picker International Inc, 1991

10. Rothendler JA, Okada RD, Wilson RA, et al: Effect of a delay in commencing imaging on the ability to detect transient thallium defects. J Nucl Med 26:880-883, 1985

11. Friedman J, VanTrain K, Maddahi J, et al: "Upward creep" of the heart. A frequent source of false-positive reversible defects during thallium-201 stress-redistribution SPECT. J Nucl Med 30:1718-1722, 1989

12. Kahn JK, Carry MM, McGhie I, et al: Quantitation of post-exercise lung thallium-201 uptake during singlephoton emission computed tomography. J Nucl Med 30:288294, 1989

13. Grunwald AM, Watson DD, Holzgrefe $\mathrm{HH}$, et al: Myocardial thallium-201 kinetics in normal and ischemic myocardium. Circulation 64:610-618, 1981

14. Okada RD, Jacobs ML, Daggett WM, et al: Thallium201 kinetics in non-ischemic canine myocardium. Circulation $65: 70-77,1982$

15. Kaul S, Chesler DA, Pohost GM, et al: Influence of peak exercise heart tate on normal thallium-201 myocardial clearance. J Nucl Med 27:26-30, 1986
16. Maddahi J, VanTrain K, Prigent F, et al: Quantitative single-photon emission computed thallium-201 tomography for detection and localization of coronary artery disease: Optimization and prospective validation of a new technique. J Am Coll Cardiol 14:1689-1699, 1989

17. Eisner $R L$, Tamas $M J$, Cloninger $K$, et al: Normal SPECT thallium-201 bull's-eye display: Gender differences. J Nucl Med 29:1902-1909, 1988

18. Ball RM, Bache RJ, Cobb FR, et al: Regional myocardial blood flow during graded treadmill exercise in the dog. J Clin Invest 55:43-51, 1975

19. DePasquale EE, Nody AC, DePuey EG, et al: Quantitative rotational thallium-201 tomography for identifying and localizing coronary artery disease. Circulation 77:316327,1988

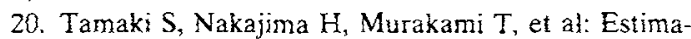
tion of infarct size by myocardial emission computed tomography with thallium-201 and its relation to creatine $\mathrm{kj}$ nase- $\mathrm{MB}$ release after myocardial infarction in man. Circulation 66:994-1001, 1982

21. Keyes JW, Leonard PF, Brody SL, et al: Myocardial infarct quantification in the dog by single-photon emission computed tomography. Circulation 58:227-232, 1978

22. Caldwell $\mathrm{JH}$, Williams $\mathrm{DL}$, Hamilton $\mathrm{GW}$, et al: Regional distribution of myocardial blood flow measured by single-photon tomography. Comparison with in vitro counting. J Nucl Med 23:490-495, 1982

23. Kirsch CM, Doliwa R, Buell U, et al: Detection of severe coronary heart disease with thallium-201: Comparison of resting single-photon emission tomography with invasive arteriography. J Nucl-Med 24:761-767, 1983

24. Hoffman EJ, Huang SC, Phelps ME: Quantitation in positron emission computed tomography. 1. Effect of object size. J Comput Assist 3:299-308, 1979

25. Tung CH, Gullberg GT, Zeng GL, et al: Non-uniform attenuation correction using simultaneous transmission and emission converging tomography. IEEE Nuclear Science (In press)

26. Datz FL: Cardiac imaging, in Handbooks in Radiology-Nuclear Medicine. Year Book Medical, Chicago, IL 1988 pp 231-271

27. Detre KM, Wright PH, Murphy ML, et al: Observer agreement in evaluating coronary angiograms. Circulation 52:979-986, 1975

28. DeRouen TA, Murray JA, Owen W: Variability in the analysis of coronary angiograms. Circulation 55:324-328, 1977

29. Hirzel HO, Senn M, Nuesch K, et al: Thallium-201 
scintigraphy in complete left bundle branch block. Am J Cardiol 53:764-769, 1984

30. Gould KL, Kelley KO, Bolson EL: Experimental validation of quantitative coronary arteriography for determining pressure-flow characteristics of coronary stenosis. Circulation 66:930-937, 1982

31. Eisner R, Churchwell A, Noever T, et al: Quantitative analysis of the tomographic thallium-201 myocardial bullseye display: Critical role of correcting for patient motion. J Nucl Med 29:91-97, 1988

32. Geckle WJ, Frank TL, Links JM, et al: Correction for patient and organ movement in SPECT: Application to exercise thallium-201 cardiac imaging. J Nucl Med 29:441450,1988
33. Rab ST, Alazraki N, Krawczynska EG: Peritoneal fluid causing inferior attenuation on SPECT thallium-201 myocardial imaging in women. J Nucl Med 29:1860-1864, 1988

34. DePuey EG, Guertler-Krawczynska E, Perkins IV, et al: Alterations in myocardial thallium-201 distribution in patients with chronic systemic hypertension undergoing single-photon emission computed tomography. Am J Cardiol 62:234-238, 1988

35. DePuey EG, Guergler-Krawczynska E, Robbins WL: Thallium-201 SPECT in coronary artery disease patients with left bundle branch block. J Nucl Med 29:1479-1485, 1988 